\title{
Identification and detection algorithm of electric energy disturbance in microgrid based on wavelet analysis and neural network
}

\author{
Songjin Liu and Dongsheng Yang
}

\author{
*Correspondence: \\ minmang2871119ga@163.com; \\ yangdongsheng@mail.neu.edu.cn \\ Northeastern University, \\ Shenyang 110819, China
}

\begin{abstract}
In the presence of power disturbance, the test accuracy of power is not good, in order to improve the performance of power testing, it is necessary to carry out the power disturbance detection design. A power disturbance detection algorithm based on wavelet analysis and neural network is proposed. The time domain and frequency domain decomposition are used to decompose the characteristic of the power disturbance signal, and the wavelet analysis method is used to improve the adaptive focusing performance of the power disturbance signal. The one-dimensional power disturbance function is mapped to the two-dimensional function of time scale and time shift by continuous wavelet transform, and the higher-order spectral characteristic quantity of the power disturbance signal is extracted, and the extracted characteristic quantity is automatically classified by neural network. The optimal identification and detection of power disturbance are realized. The simulation results show that the algorithm has higher accuracy and higher recognition ability, which improves the accurate probability of power disturbance detection and the anti-disturbance ability of power test.
\end{abstract}

Keywords: Wavelet analysis, Neural network, Power disturbance, Recognition, Detection

\section{Introduction}

With the continuous expansion of the scale of the State Grid, people pay more attention to the real-time fault monitoring and protection of the smart grid [1]. Combined with the development of the power measurement technology and the software calculation, the smart grid computer network communication technology is assembled $[2,3]$. The smart grid computer network based on computer technology and wireless communication is widely used in the field of power network control, and plays an important role in power transmission, power transmission, distribution and so on $[4,5]$. In the process of power transmission and distribution, electric energy testing is needed to improve the accurate measurement and scheduling ability of electric energy [6]. However, the existence of power disturbance seriously affects the accuracy of power test. It is necessary to optimize the identification and detection of power disturbance, improve the accuracy of power measurement, and study the accurate

(c) The Author(s) 2021. Open Access This article is licensed under a Creative Commons Attribution 4.0 International License, which permits use, sharing, adaptation, distribution and reproduction in any medium or format, as long as you give appropriate credit to the original author(s) and the source, provide a link to the Creative Commons licence, and indicate if changes were made. The images or other third party material in this article are included in the article's Creative Commons licence, unless indicated otherwise in a credit line to the material. If material is not included in the article's Creative Commons licence and your intended use is not permitted by statutory regulation or exceeds the permitted use, you will need to obtain permission directly from the copyright holder. To view a copy of this licence, visit http:// creativecommons.org/licenses/by/4.0/. 
detection method of power disturbance. It has great significance in power output measurement and power dispatch management [7].

Traditionally, there are time domain analysis, frequency domain analysis, time-frequency distribution feature detection method, statistical feature analysis method and high-order spectral feature extraction method for power disturbance detection $[8,9]$. Combined with the method of extracting impulse response of time-frequency pulse, the signal testing is realized, and the performance of signal detection is improved, and some research results are obtained. Among them, in reference [10], a power disturbance detection technique based on adaptive lifting wavelet transform is proposed, which uses time-domain and frequency-domain decomposition to decompose the signal characteristic $[11,12]$. Signal detection and output testing of power disturbance are realized by using high resolution echo detection technology, but the anti-jamming ability and signal to noise ratio of signal output are not strong [13]. An energy disturbance signal detection technique based on energy spectral density function feature clustering is proposed [14]. The method of information fusion is used to detect the wideband feature of the signal and to realize the weak correlation feature test and signal detection of the power disturbance signal. However, this method is greatly influenced by Nyquist sampling time interval, and the accuracy of detecting power disturbance with high power interference is not high [15].

In order to solve the above problems, this paper proposes a power disturbance detection algorithm based on wavelet analysis and neural network [16]. The time domain and frequency domain decomposition are used to decompose the characteristic of the power disturbance signal, and the wavelet analysis method is used to improve the adaptive focusing performance of the power disturbance signal [17, 18]. The one-dimensional power disturbance function is mapped to the two-dimensional function of time scale and time shift by continuous wavelet transform, and the higher-order spectral characteristic quantity of the power disturbance signal is extracted, and the extracted characteristic quantity is automatically classified by neural network [19]. The optimal identification and detection of power disturbance are realized. Finally, a simulation experiment is carried out to demonstrate the superior performance of the proposed method in improving the decomposition power of the energy characteristic of power disturbance detection [20].

The specific contributions of this paper include:

1. A power interference detection algorithm based on wavelet analysis and neural network is proposed.

2. Use time domain and frequency domain decomposition to decompose the characteristics of power interference signals, and use wavelet analysis method to improve the adaptive focusing performance of power interference signals.

3. The one-dimensional power interference function is mapped to the two-dimensional function of time scale and time shift through continuous wavelet transform, and the high-order spectral feature quantity of the power interference signal is extracted, and the extracted feature quantity is automatically classified.

4. Realize the best identification and detection of power interference. 
The rest of this paper is organized as follows. Section 2 discusses Power disturbance signal model and characteristic decomposition, followed by the methods is discussed in Sect. 3. The experiment is discussed in Sect. 4. Section 5 concludes the paper with summary and future research directions.

\section{Power disturbance signal model and characteristic decomposition}

\subsection{Power disturbance signal model}

In order to detect and identify the power disturbance accurately [21], the signal model should be constructed first, and a wideband echo model is given as:

$$
g(t)=\sqrt{s} f(s[t-\tau])
$$

In which, $f(t)$ is the transmission signal of power disturbance, $s=(c-v) /(c+v)$, which is called time scale factor, which is called scale. $\tau=2 R / c$, it represents the expansion and expansion of power disturbance signal. $R$ is the radial distance between the power output node and the receiving node, $\sqrt{s}$ is the normalized factor [22].

The one-dimensional function of power disturbance is mapped to the two-dimensional function of time scale $a$ and time shift $b$ by continuous wavelet transform. The square integrable function $y(t)$ is used for the continuous wavelet transform, the mother wavelet $\psi(t)$ is obtained [23].

$$
W_{\psi} y(a, b)=\left\langle y, \psi_{a, b}\right\rangle=\int_{-\infty}^{+\infty} y(t) \frac{1}{\sqrt{|a|}} \psi^{*}\left(\frac{t-b}{a}\right) \mathrm{d} t
$$

By the strict periodic frequency domain decomposition [24], the time domain and frequency domain are combined to obtain the frequency domain characteristic quantity of the power disturbance signal:

$$
\psi_{a, b}(t)=[U(a, b) \psi(t)]=\frac{1}{\sqrt{|a|}} \psi\left(\frac{t-b}{a}\right)
$$

In which, $U(a, b)$ is the affine group unitary transformation for the mother wavelet [25], and the factor $1 / \sqrt{|a|}$ ensures the energy normalization of the unitary transformation.

\subsection{Characteristic decomposition of power disturbance signals}

Time domain and frequency domain decomposition are used to decompose the characteristic of power disturbance signal, and wavelet analysis method is used to improve the adaptive focusing performance of power disturbance signal [26]. The power disturbance $f(t)$ is taken as the mother wavelet function $\psi(t)$, and the variables are replaced, $a=1 / s, b=\tau$, rewritten formula (3):

$$
f_{s, \tau}(t)=[U(1 / s, \tau) f(t)]=\sqrt{|s|} f(s(t-\tau))
$$

Formula (4) clearly shows that the power disturbance model is closely related to the wavelet transform. If the power disturbance signal is taken as the mother wavelet function, the wavelet transform has distinct physical meaning and is a natural 
mathematical tool for wideband signal processing $[27,28]$. The linear time-frequency representation model of power disturbance signal is obtained as follows:

$$
X(u)=\sqrt{\frac{1-j \cot \alpha}{2 \pi}} \int_{-\infty}^{+\infty} x(t) \exp \left[j \frac{t^{2}+u^{2}}{2} \cot \alpha-j \csc \alpha\right] \mathrm{d} t
$$

In which, $x(t)$ denotes the time-frequency joint distribution characteristic function of the signal, and $\alpha$ is the time-window function [29].

\section{Methods}

\subsection{Wavelet analysis of power disturbance}

Based on the characteristic decomposition of power disturbance signal in time domain and frequency domain, the optimal detection algorithm of power disturbance is designed. In this paper, the power disturbance identification and detection algorithm based on wavelet analysis and neural network is proposed. Combined with wavelet analysis method to improve the adaptive focusing performance of power disturbance signal, the one-dimensional power disturbance function is mapped to twodimensional function of time scale and time shift by continuous wavelet transform, and the high power disturbance signal is extracted [30]. High-order spectral characteristics are extracted, the rectangular envelope signal of power disturbance is:

$$
u(t)=\frac{1}{\sqrt{T}} \operatorname{rect}\left(\frac{t}{T}\right) \exp \left\{-j\left[2 \pi K \ln \left(1-\frac{t}{t_{0}}\right)\right]\right\}
$$

In which, $\operatorname{rect}(t)=1,|t| \leq 1 / 2$. The frequency modulation law of power disturbance signal is a hyperbolic function, that is:

$$
f_{i}(t)=\frac{K}{t_{0}-t} \quad|t| \leq \frac{T}{2}
$$

In which, $K=T f_{\max } f_{\min } / B, t_{0}=f_{0} T / B f_{0}$ is the arithmetic centre frequency of power disturbance [31], $f_{\min }, f_{\max }$ are the lowest and highest frequency of power disturbance. The transform of small wave of electric energy disturbance signal into:

$$
\begin{aligned}
W_{u} u(a, b)= & e^{j 2 \pi K \ln a} \times \frac{K}{\sqrt{a}}\left\{\left[\frac{a e^{\frac{j 2 \pi f_{\min }}{a}\left(b-b_{a}\right)}}{f_{\min }}-\frac{e^{j 2 \pi f_{\max }\left(b-b_{a}\right)}}{f_{\max }}\right]\right. \\
& \left.+j 2 \pi\left(b-b_{a}\right)\left[E i\left(j 2 \pi f_{\max }\left(b-b_{a}\right)\right)-E i\left(\frac{j 2 \pi f_{\min }}{a}\left(b-b_{a}\right)\right)\right]\right\}
\end{aligned}
$$

In which, $b_{a}=(1-a)\left(\frac{1}{a f_{\max }}-\frac{T}{2}\right), E i(\bullet)$ represents a time window weighting.

\subsection{Feature extraction and neural network classification and recognition}

The one-dimensional power disturbance function is mapped to the two-dimensional function of time scale and time shift by continuous wavelet transform, and the higherorder spectral characteristic of power disturbance signal is extracted as: 


$$
\theta(t)=2 \pi \int_{-T / 2}^{t / a}\left(\frac{K}{t_{0}-t^{\prime}}\right) \mathrm{d} t^{\prime}=-2 \pi K \ln \left(1-\frac{t}{a t_{0}}\right)+\theta_{0}
$$

In which, $\theta_{0}=-2 \pi K \ln \left(1+\frac{T}{2 t_{0}}\right)$, the instantaneous frequency of electrical disturbance is:

$$
f_{i_{a}}(t)=\frac{1}{2 \pi} \frac{\mathrm{d} \theta}{\mathrm{d} t}=\frac{K}{a t_{0}-t}=\frac{K}{t_{0}-t+(a-1) t_{0}}
$$

A neural network classifier is used to classify and identify the extracted features. The neural network model is shown in Fig. 1.

The neural network in Fig. 1 is a multivariable controlled object with m input and n output. Using a PIDNN multivariable classifier with $m$ output, the output of each neuron in the hidden layer is obtained:

$$
x_{s j}^{\prime}(k)= \begin{cases}1, & u_{s j}^{\prime}(k)>1 \\ u_{s j}^{\prime}(k), & -1 \leq u_{s j}^{\prime}(k) \leq 1 \\ -1, & u_{s j}^{\prime}(k)<-1\end{cases}
$$

Perturbation eigenvalue of input is:

$$
f_{i_{a}}(t)=f_{i}\left[t-(a-1) t_{0}\right]
$$

The identification parameter is:

$$
b_{m}=t_{0}(1-a)
$$

Then:

$$
f_{i_{a}}(t)=f_{i}\left(t+b_{m}\right)
$$

The feature classification of power disturbance is carried out on the time scale. The scale-time translation model of power disturbance feature classification is shown in Fig. 2.

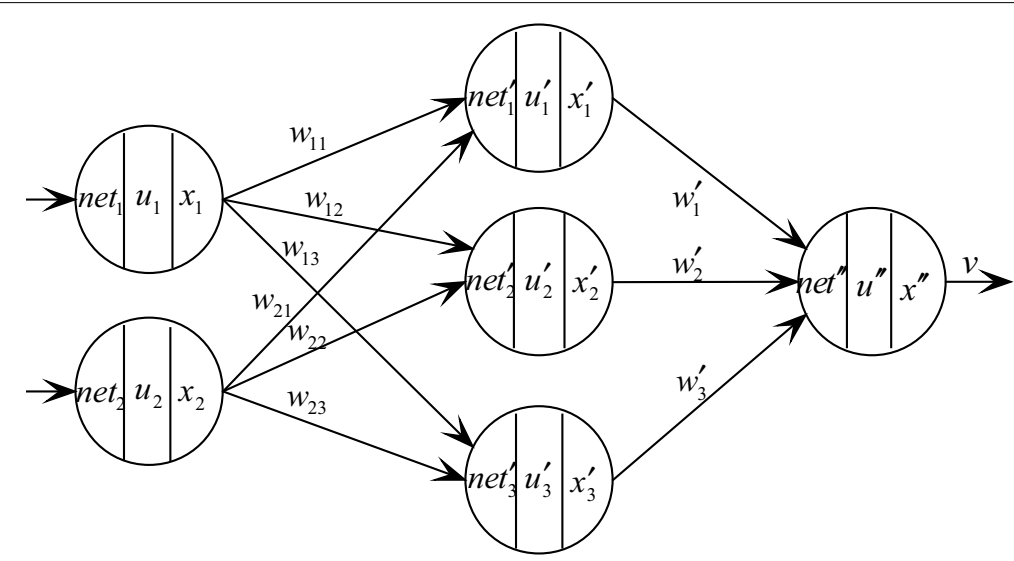

Fig. 1 Neural network model 


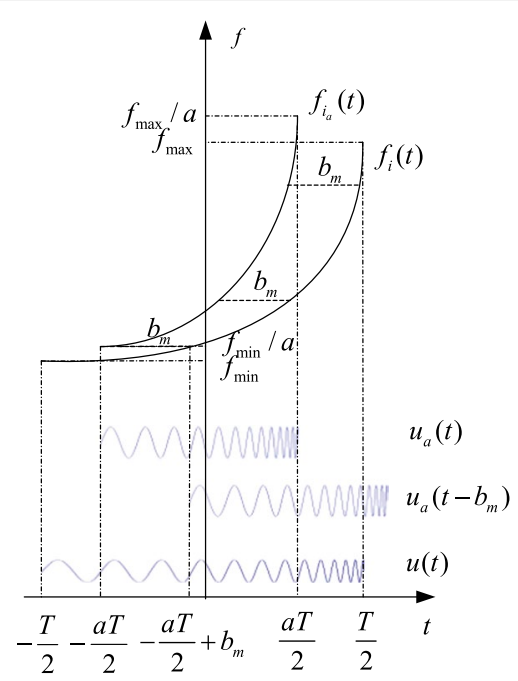

Fig. 2 Scale scaling and time translation model for feature classification of power disturbance

In Fig. 2, by wavelet analysis and neural network classification, the time domain components of power disturbance detection are obtained as follows:

$$
u_{a}\left(t-b_{m}\right)=\frac{1}{\sqrt{a}} u(t) \quad-\frac{a T}{2}+b_{m}<t<\frac{a T}{2}+b_{m}
$$

Write the output of the power disturbance detection component in a unified form:

$$
W_{u} u\left(a, b_{m}\right)=\frac{1}{\sqrt{a}}\left(\frac{a+1}{2}-\frac{|a-1|}{B / f_{0}}\right)
$$

In which, the characteristics of the extracted feature are classified by neural network automatically to realize the optimal identification and detection of power disturbance.

\section{Experiment}

In order to test the application performance of this algorithm in power disturbance detection, the simulation experiment is carried out. The experiment is designed with Matlab simulation tool. The time width of power disturbance signal is $0.01 \mathrm{~s}$, the local stationary bandwidth is 0.4 , and the energy normalization are obtained. The signal model output of the power disturbance is shown in Fig. 3.

Signal model of power disturbance is used to test objective. The feature extraction of high-order spectrum is carried out, and the time-frequency decomposition is carried out by wavelet transform, combining neural network classifier for feature classification, the feature extraction and detection output are shown in Fig. 4.

It shows that the beam focusing ability of power disturbance detection using this method is good, which shows that the disturbance detection has strong anti-jamming ability. Different methods are used to test the performance of power disturbance detection, and the results are shown in Fig. 5. The analytical Fig. 5 shows that the accurate probability of detecting and identifying power disturbance by this method is high. 


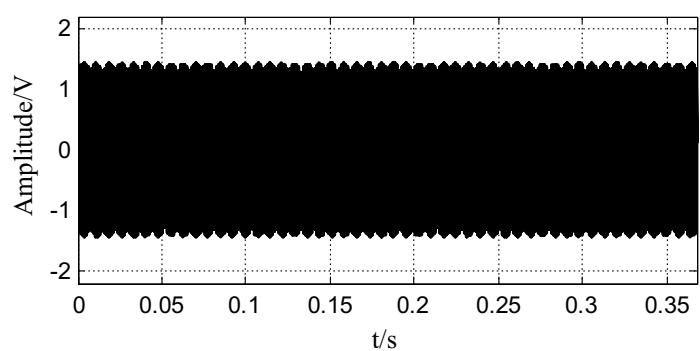

Fig. 3 Signal model of power disturbance

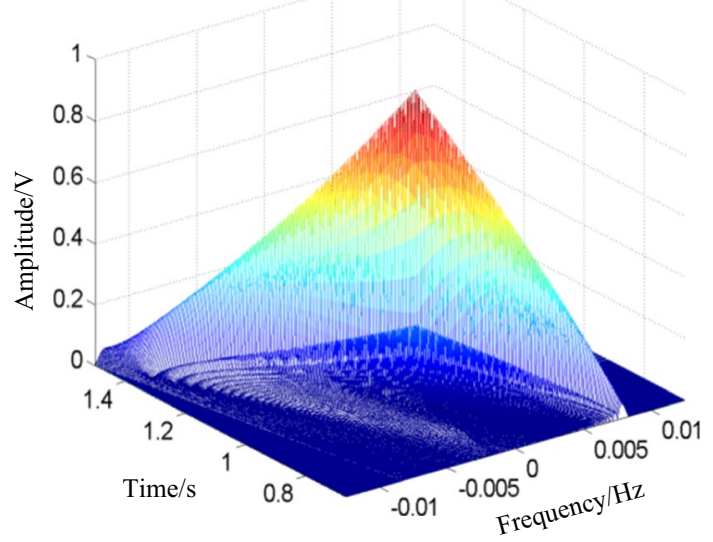

Fig. 4 Wavelet transform of power disturbance signal

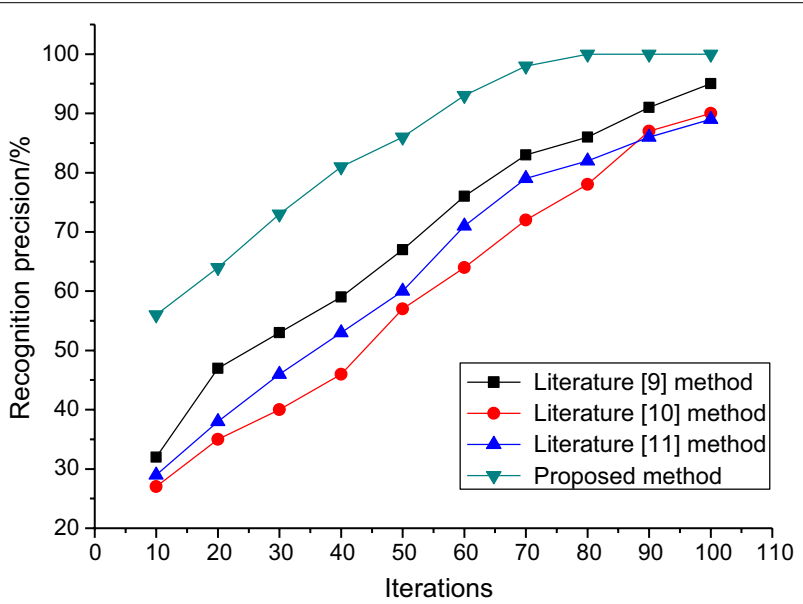

Fig. 5 Performance comparison of detection and recognition

\section{Results and discussion}

In order to improve the performance of electric energy testing, it is necessary to design the detection of power disturbance. A detection algorithm of power disturbance based on wavelet analysis and neural network is proposed. Time domain and frequency domain decomposition are used to decompose the characteristic of power disturbance 
signal, and wavelet analysis method is used to improve the adaptive focusing performance of power disturbance signal. One-dimensional power disturbance function is mapped to time by continuous wavelet transform. The two-dimensional function of scale and time translation is used to extract the high-order spectral characteristic of the power disturbance signal. The neural network is used to classify the extracted feature to realize the optimal identification and detection of the power disturbance. The simulation results show that the algorithm has higher accuracy and higher recognition ability, which improves the accurate probability of power disturbance detection and the antidisturbance ability of power test.

Abbreviation

PIDNN: Proportional-Integral-Derivative Neural Network.

Acknowledgements

National Natural Science Foundation of China (approval number: U1908217).

Authors' contributions

Songjin Liu is responsible for the collection of experimental data, and Dongsheng Yang is responsible for the writing of the paper. Both authors read and approved the final manuscript.

Funding

National Natural Science Foundation of China (approval number: U1908217).

Availability of data and materials

Data sharing not applicable to this article as no datasets are generated or analyzed during the current study.

Ethics approval and consent to participate

This article does not contain any studies with human participants or animals performed by any of the authors.

\section{Consent for publication}

All authors agree to submit this version and claim that no part of this manuscript has been published or submitted elsewhere.

\section{Competing interests}

All authors declare that they have no conflict of interest.

Received: 31 October 2020 Accepted: 12 January 2021

Published online: 12 February 2021

\section{References}

1. J. Pan, Signal detection in strong chaotic interference based on RBF neural network. Comput. Simul. 5, 136-139 (2010)

2. Y. Cheng, W. Yang, Study on pseudo-noise code tracking method for compressive domain direct sequence tracking telemetry and command communication signals. JEIT 37(8), 2028-2032 (2015)

3. Z. Wu, B. Pei, The detection of LDoS attack based on the model of small signal. Chin. J. Electron. 39(6), 1456-1460 (2011)

4. H. Liang, J. Han, Clustering and sorting radar signals based on multi-wavelet packets characteristics of bispectrum. Acta Photonica Sinica 43(3), 0307001 (2014)

5. C. Sun, C. Yang, S. Fan et al., Energy efficient distributed clustering consensus filtering algorithm for wireless sensor networks. Inf. Control 44(3), 379-384 (2015)

6. C. Shang, P. Wang, Z. Han et al., Feature-level fusion recognition algorithm based on analogy decision tree classification. Control Decis. 31(06), 1009-1014 (2016)

7. G. Hu, H. Liang, Q. Xu, Research on chaotic feature extraction of ship radiated noise. Comput. Simul. 28(2), 22-24 (2011)

8. I.A. Kareem, M.G. Duaimi, Improved accuracy for decision tree algorithm based on unsupervised discretization. Int. J. Comput. Sci. Mobile Comput. 3(6), 176-183 (2014)

9. Y. Gao, X. Zhao, Adaptive combination algorithm and its modified scheme for blind source separation. J. Electron. Inf. Technol. 33(5), 1243-1247 (2011)

10. P. Frihauf, M. Krstic, T. Basar, Nash equilibrium seeking in noncooperative games. IEEE Trans. Autom. Control 57(5), 1192-1207 (2012)

11. M.R. Hesamzadeh, D.R. Biggar, Computation of extremal-Nash equilibria in a single-stage MILP. IEEE Trans. Power Syst. 27(3), 1706-1707 (2012)

12. Y. Yang, C. Xia, W. Tong, Estimation of the time varying TDOA and FDOA in GEO-LEO dual-satellites location system. Signal Process. 28(10), 1465-1474 (2012) 
13. T.C. Nguyen, W. Shen, Z. Luo et al., Novel Data Integrity Verification Schemes in Cloud Storage (Springer, Cham, 2015), pp. $115-125$

14. X. Yi, S. Peng, S. Luan, An approach of adaptive signal separation based on operator and locally orthogonal constraint. JEIT 37(11), 2613-2620 (2015)

15. S.G. Hao, L. Zhang, G. Muhammad, A union authentication protocol of cross-domain based on bilinear pairing Journal of Software $\mathbf{8}(5), 1094-1100$ (2013)

16. C.C. Tanner, J.P.S. Sukias, M.P. Upsdell, Substratum phosphorus accumulation during maturation of gravel-bed constructed wetlands. Water Sci. Technol. 40, 147-154 (1999)

17. H.C. Tee, P.E. Lim, C.E. Seng, M.A. Nawi, Newly developed baffled subsurface-flow constructed wetland for the enhancement of nitrogen removal. Bioresour. Technol. 104, 235-242 (2012)

18. C. Vohla, M. Kõiv, H.J. Bavor, F. Chazarenc, U. Mander, Filter materials for phosphorus removal from wastewater in treatment wetlands-a review. Ecol. Eng. 37, 70-89 (2011)

19. J. Vymazal, Horizontal sub-surface flow and hybrid constructed wetlands systems for wastewater treatment. Ecol. Eng. 25, 478-490 (2005)

20. J. Vymazal, L. Kröpfelová, Removal of organics in constructed wetlands with horizontal sub-surface flow: a review of the field experience. Sci. Total Environ. 407, 3911-3922 (2009)

21. S. Warneke, L.A. Schipper, M.G. Matiasek, K.M. Scow, S. Cameron, D.A. Bruesewitz, I.R. McDonald, Nitrate removal, communities of denitrifiers and adverse effects in different carbon substrates for use in denitrification beds. Water Res. 45, 5463-5475 (2011)

22. Y. Wen, Y. Chen, N. Zheng, D. Yang, Q. Zhou, Effects of plant biomass on nitrate removal and transformation of carbon sources in subsurface-flow constructed wetlands. Bioresour. Technol. 101, 7286-7292 (2010)

23. H. Wu, J. Fan, J. Zhang, H.H. Ngo, W. Guo, Z. Hu et al., Optimization of organics and nitrogen removal in intermittently aerated vertical flow constructed wetlands: effects of aeration time and aeration rate. Int. Biodeter. Biodegr. 113 139-145 (2016)

24. H. Wu, J. Fan, J. Zhang, H.H. Ngo, W. Guo, Z. Hu et al., Decentralized domestic wastewater treatment using intermittently aerated vertical flow constructed wetlands: impact of influent strengths. Bioresour. Technol. 176, 163-168 (2015)

25. H. Wu, J. Zhang, H.H. Ngo, W. Guo, Z. Hu, S. Liang et al., A review on the sustainability of constructed wetlands for wastewater treatment: design and operation. Bioresour Technol 175, 594-601 (2015)

26. H. Yin, X. Yan, X. Gu, Evaluation of thermally-modified calcium-rich attapulgite as a low-cost substrate for rapid phosphorus removal in constructed wetlands. Water Res. 115, 329-338 (2017)

27. C. Zhang, Q. Yin, Y. Wen, W. Guo, C. Liu, Q. Zhou, Enhanced nitrate removal in self-supplying carbon source constructed wetlands treating secondary effluent: the roles of plants and plant fermentation broth. Ecol. Eng. 91, 310-316 (2016)

28. D.Q. Zhang, R.M. Gersberg, K. Tansoon, Constructed wetlands in china. Ecol. Eng. 35, 1367-1378 (2009)

29. M. Zhang, L. Zhao, C. Mei, L. Yi, G. Hua, Effects of plant material as carbon sources on tn removal efficiency and N2O flux in vertical-flow-constructed wetlands. Water Air Soil Poll. 225, 2181 (2014)

30. X. Zhou, L. Jia, C. Liang, L. Feng, R. Wang, H. Wu, Simultaneous enhancement of nitrogen removal and nitrous oxide reduction by a saturated biochar-based intermittent aeration vertical flow constructed wetland: effects of influent strength. Chem. Eng. J. 334, 1842-1850 (2017)

31. X. Zhou, X. Wang, H. Zhang, H. Wu, Enhanced nitrogen removal of low $\mathrm{c} / \mathrm{n}$ domestic wastewater using a biocharamended aerated vertical flow constructed wetland. Bioresour. Technol. 241, 269-275 (2017)

\section{Publisher's Note}

Springer Nature remains neutral with regard to jurisdictional claims in published maps and institutional affiliations.

\section{Submit your manuscript to a SpringerOpen ${ }^{\circ}$ journal and benefit from:}

- Convenient online submission

- Rigorous peer review

- Open access: articles freely available online

- High visibility within the field

- Retaining the copyright to your article

Submit your next manuscript at $\boldsymbol{\nabla}$ springeropen.com 